\title{
Das andere Bali I: Gemeinschaftsland und Ältestenräte in den bali aga-Gemeinden von Kintamani
}

\section{Die bali aga im balinesischen Kontext}

Im balinesischen Weltbild stehen den überweltlichen Kräften niederweltliche gegenüber, den bergwärts gelegenen Ursprungstempeln meerwärts gerichtete Tempel der Niederwelt bzw. des Todes, den Gottheiten dämonische Wesen und dem Sonnenaufgang der Sonnenuntergang (vgl. LEEMANN 1976). Diesen und anderen komplementären Aspekten ein und desselben Weltbildes steht wiederum ein von Forschung und Tourismus geprägtes Balibild gegenüber, das vor allem die lichten Aspekte betont: Kunst und Kultur, Klänge und Farben, Tänze und Tempel Südbalis, dem balinesischen «heartland» (C. GEERTZ 1980: 47). Ausgespart bleiben die entstehende panindonesische Gesellschaft in den urbanen Zentren (vgl. TARNUTZER 1993) sowie die kargere und wahrscheinlich ursprünglichere Kultur und Gesellschaft in den Berggebieten der Insel. Dieser Artikel handelt von diesem anderen, unbekannteren Bali, und zwar von den Bergdörfern der Region Kintamani, die zum altbalinesischen oder bali aga-Gebiet gehören.

Von ihren Strandhotels aus besuchen viele Touristen Kintamani in einem Tagesausflug. Von der Straße, die auf dem südlichen Rand der Baturcaldera entlang führt, bewundern sie die spektakuläre Aussicht auf den aktiven Schichtvulkan Gunung Batur, die Lavafelder in Innern der über $10 \mathrm{~km}$ weiten Caldera und auf die höchsten Vulkane Balis. Vor den Leuten dort aber wird gewarnt, seien sie doch die aufdringlichsten Verkäufer der Insel und immer bereit, Touristen übers $\mathrm{Ohr}$ zu hauen und auch sonst eher unansehnlich und hinterwäldlerisch.' Selbst Margaret Mead hat 1936 die Wahl ihres wenige Kilometer südlich der Caldera gelegenen Untersuchungsdorfes Bayung Gede damit begründet, daß eine Schilddrüsendefizienz die Handlungen der Einheimischen derart verlangsame, daß kulturelle Muster leichter erkennbar seien als in komplexeren Orten in Balis Süden. Brieflich hielt sie fest, daß Bayung Gede "the lowest and dourest stratum» der balinesischen Kultur repräsentiere (HOWARD 1985: 186)2 und der gut 20 Jahre nach Margaret Mead auf Bali arbeitende, weltweit bekannte Kulturanthropologe C. GEERTZ hält fest, daß die Bedeutung der bali aga-Dörfer «marginal in the extreme» sei (1959: 1012). LANSING (1983: $113 \mathrm{f}$.) bezeichnet sie als anomal (anomalous) im Gegensatz zu den normalen (ordinary) Dörfern. An anderer Stelle erklärt wiederum C. GEERTZ die bali aga-Dörfer zu geodeterministisch begründeten, vernachlässigbaren Spezialfällen: "To my mind, it is not the heartland villages, in which virtually the entire population seems to have lived for centuries, which are atypical, eccentric outcomes of the play of special circumstances; it is the remoter ones, scattered along the margins of the heartland" (1980:47). So erstaunt es nicht, daß die postkoloniale Forschung sich auf die südbalinesische, vom vergangenen ostjavanischen Hindureich Majapahit mitbeeinflußte Kultur und Gesellschaft konzentriert. Aus dieser Perspektive des balinesischen "heartland", zu dem C. GEERTZ ein vielbeachtetes Buch verfaßt hat, dem die zitierte Stelle entnommen ist, mag diese Bewertung eine gewisse Berechtigung haben. Richtiger aber ist, daß - wie schon die niederländischen Forscher der kolonialen Periode feststellten - die räumliche Verbreitung der altbalinesischen oder bali aga-Kultur sich auf große Teile des Nordens, des Ostens und des gebirgigen Innerns der Insel erstreckt (KORN 1932: 77 ff.). Zwar sind die bali aga eine Minderheit, aber durchaus - auch quantitativ - von einer gewissen Bedeutung. ${ }^{3}$

Trotzdem sind die bali aga in den letzten Dekaden wenig im eigentlichen Sinne erforscht worden, und die altbalinesische Kultur und Gesellschaft wird oft nur unter einem doppelt exotischen Blickwinkel wahrgenommen: Was im Kontrast zum - vom westlichen Standpunkt aus betrachtet - anderen oder exotischen Südbali wiederum anders oder fremd erscheint, wird registriert. So ist der Begriff bali aga oder altbalinesisch manchmal fälschlicherweise mit "vorhinduistisch" gleichgesetzt worden. ${ }^{4}$ Hinduistische und buddhistische Einflüsse sind für Bali seit dem 8. Jahrhundert belegt, wahrscheinlich aber älteren Datums. In den altbalinesischen Gebieten ist einzig die letzte, durch das ostjavanische Reich Majapahit im 14. Jahrhundert ausgelöste Hinduisierungsphase ohne prägenden Einfluß geblieben. Die bali aga-Dörfer wußten in der Folge ihre Identität gegenüber den hindujavanisch beeinflußten Fürstenhäuser der balinesischen Tieflandgebiete weitgehend zu bewahren. So wohnen in den bali aga-Gemeinden kaum Angehörige höherer Kasten, Verstorbene werden nicht kremiert, sondern begraben und im Dorf Trunyan sogar ausgesetzt. Als Elemente altindonesischer Gesellschaftsordnung und Ausdruck von Egalität in diesen Dörfern gelten insbesondere das

Samuel Wälty, dipl. Geograph, Geographisches Institut der Universität Zürich-Irchel, Winterthurerstr. 190, 8057 Zürich 
Gemeinschaftsland, das im Nutzrecht parzellenweise an Dorfmitglieder vergeben wird, sowie der auf dem Senioritätsprinzip beruhende Ältestenrat, in dem der Aufstieg in der Rangordnung einzig durch das Alter bestimmt wird. So beschreibt HOWE (1989: 50) die soziale Organisation dieser altbalinesischen oder bali aga-Dörfer als "based on seniority and an ideology of egalitarianism». Diese beiden Institutionen in den Bergdörfern von Kintamani, das Gemeinschaftsland und der Ältestenrat, stehen im Zentrum der folgenden Ausführungen. Die beiden Institutionen werden vorgestellt, und anschließend wird erörtert, inwiefern Gemeinschaftsland und Ältestenrat als Merkmale einer egalitären Sozialordnung betrachtet werden können. Schließlich werden anhand des Gemeinschaftslandes einige Aspekte sozialen Wandels aufgezeigt. Vorgängig aber wird die «extrem marginale» Untersuchungsregion Kintamani vorgestellt, auf daß sie ein wenig vom Rande wegrücken möge. Dabei wird auch die raum-zeitliche Ausdehnung der Institutionen Gemeinschaftsland und Ältestenrat gestreift. Andere Aspekte altbalinesischer Sozialordnung werden hier ebenso ausgeklammert wie ein Vergleich mit den Tieflandgebieten (vgl. dazu den Artikel A. Leemann in dieser Nummer). Die Daten stammen größtenteils aus einer 1986 durchgeführten Untersuchung in den 48 administrativen Gemeinden des Bergbezirks Kintamani. ${ }^{5}$

\section{Kintamani}

Der Bezirk (kecamatan) Kintamani im östlichen zentralen Bergland von Bali umfaßt eine Fläche von $367 \mathrm{~km}^{2}$ (vgl. Karte). Die Siedlungskerne der von 64000 Menschen bewohnten 48 administrativen Gemeinden liegen zwischen 950 und 1550 Metern über Meer, der Gipfel des höchsten Berges erreicht 2152 Meter. Der Bezirk Kintamani mit dem Hauptort gleichen Namens ist Teil des Distrikts (kabupaten) Bangli, der als einziger der acht Distrikte der Provinz Bali nicht ans Meer grenzt. Kintamani ist dünner besiedelt als die Tiefländer. Nur 2,5\% der 2,6 Millionen Balinesen wohnen auf 8\% der insgesamt $5633 \mathrm{~km}^{2}$ umfassenden Inselfläche (1984). Eine etwas weniger prosaische Annäherung erlaubt die Bedeutung von Kintamani im Buddhismus. Denn cintamani ist ein von Flammen umranktes Juwel, das sich bei gewissen Buddhafiguren an der Spitze einer hohen Haarkrone befindet $^{6}$, ähnlich wie sich Kintamani mit der atemberaubenden, von feuerspeienden Bergen umrankten Baturcaldera als krönendem Juwel im Zentrum, auf dem Scheitel einer schönen Insel befindet. Auf der Außenseite der Caldera fallen die Hänge steil gegen das nahe Meer im Norden und Osten, etwas weniger steil gegen das südliche Tiefland und fast schon sanft gegen das CaturHochland im Westen ab. Jahreszeitlich vorherrschende Winde sind der regenbringende Westmonsun und der trockene Ostmonsun. Zahlreiche Bäche entspringen somit vor allem den West- und Südflanken der Caldera und vereinigen sich zu Flüssen, die sich tief in die relativ lokkeren vulkanischen Ablagerungen einkerben. Die Sied- lungen liegen deshalb im Süden und Südwesten des Bezirks auf den flachen Hangrücken zwischen zwei Wasserläufen, im trockeneren Norden auf Spornen. Anders als im Tiefland, wo die Höfe in lockerer Anordnung mehrere Gebäude und Hausgärten umfassen, stehen die kleinen Häuser dicht aneinandergedrängt in geschlossenen Bergdörfern, die vielfach noch bis in dieses Jahrhundert von mit Dornen gespickten Zäunen gesichert waren. Auf den an die Siedlungen anschließenden Äckern werden Trockenreis, Mais, verschiedene Bohnenarten, Maniok, Bataten, Gemüse und manchmal auch Erdnüsse angebaut. Die die Flüsse säumenden Steilhänge liefern Holz und Bambus, Viehfutter und Wildpflanzen.

Einige Hinweise auf frühere Formen des Landeigentums vermitteln in Kupferplatten geritzte Urkunden (prasasti) aus dem 9. bis 14. Jahrhundert, welche etwa die Stiftung von Klöstern oder die Rechte und Pflichten von Dörfern festhalten (vgl. GORIS 1954). Land scheint bereits damals gehandelt worden zu sein. So haben die Dörfer Buahan im Jahr 1025 und Sukawana im Jahr 1200 den jeweiligen Fürsten Land abgekauft. ${ }^{?}$ In verschiedenen Urkunden enthaltene erbrechtliche Bestimmungen lassen darauf schließen, daß mindestens teilweise individuelle Nutz-, wahrscheinlich aber sogar Eigentumsrechte vererbt wurden. ${ }^{8}$ Damit scheint sicher, daß schon in jener Periode Land nicht ausschließlich als Gemeinschaftseigentum betrachtet wurde. Die in den Urkunden erwähnten Landnutzungsarten entsprechen ziemlich genau der heute üblichen Kategorisierung: bewässertes Ackerland, Ackerland im Trockenfeldbau, Weideland, (Baum-)Gärten. ${ }^{9}$ Bei der Vergabe der Urkunden sind die Dörfer durch ihre Ältesten vertreten, die vereinzelt ab etwa der Jahrtausendwende noch heute geläufige Titel tragen. In der zweitletzten erhaltenen Urkunde aus dem Jahr 1384 erscheint erstmals eine Rang- und Titelfolge, die der heute üblichen entspricht (vgl. KORN 1932: 20).

Während der folgenden Jahrhunderte wird über Kintamani fast nichts bekannt. Zu Beginn des 19. Jahrhunderts ist Kintamani Teil des Fürstentums Bangli. Noch vor der Kolonialisierung von Bangli wird in Kintamani von Chinesen Kaffee für den Export angebaut, ein erstes Anzeichen für die Integration des peripheren Kintamani in den Weltmarkt. Nach der niederländischen Eroberung von Südbali wird Bangli als eines der letzten balinesischen Fürstentümer 1918 der sogenannt direkten Kolonialverwaltung unterstellt und die Gemeinden Kintamanis in eine staatliche Administration integriert. Seither wird zwischen administrativen Dörfern (desa dinas) und traditionellen Dörfern (desa adat) unterschieden, die je unterschiedliche Funktionen erfüllen, Ämter und manchmal auch Grenzen aufweisen. ${ }^{10}$ Ein desa dinas kann ein desa adat, einen Teil davon oder auch mehrere Adat dörfer umfassen. Öfters als in anderen Teilen Balis sind desa dinas und desa adat in Kintamani gebietsmäßig deckungsgleich. Auf der administrativen Ebene besteht der Bezirk aus 48, auf der traditionellen aus 53 Dörfern. Von den in den 20er Jahren eingeführten Steuern war die Landsteuer vor dem Freikauf von Frondiensten und der Schlacht- 


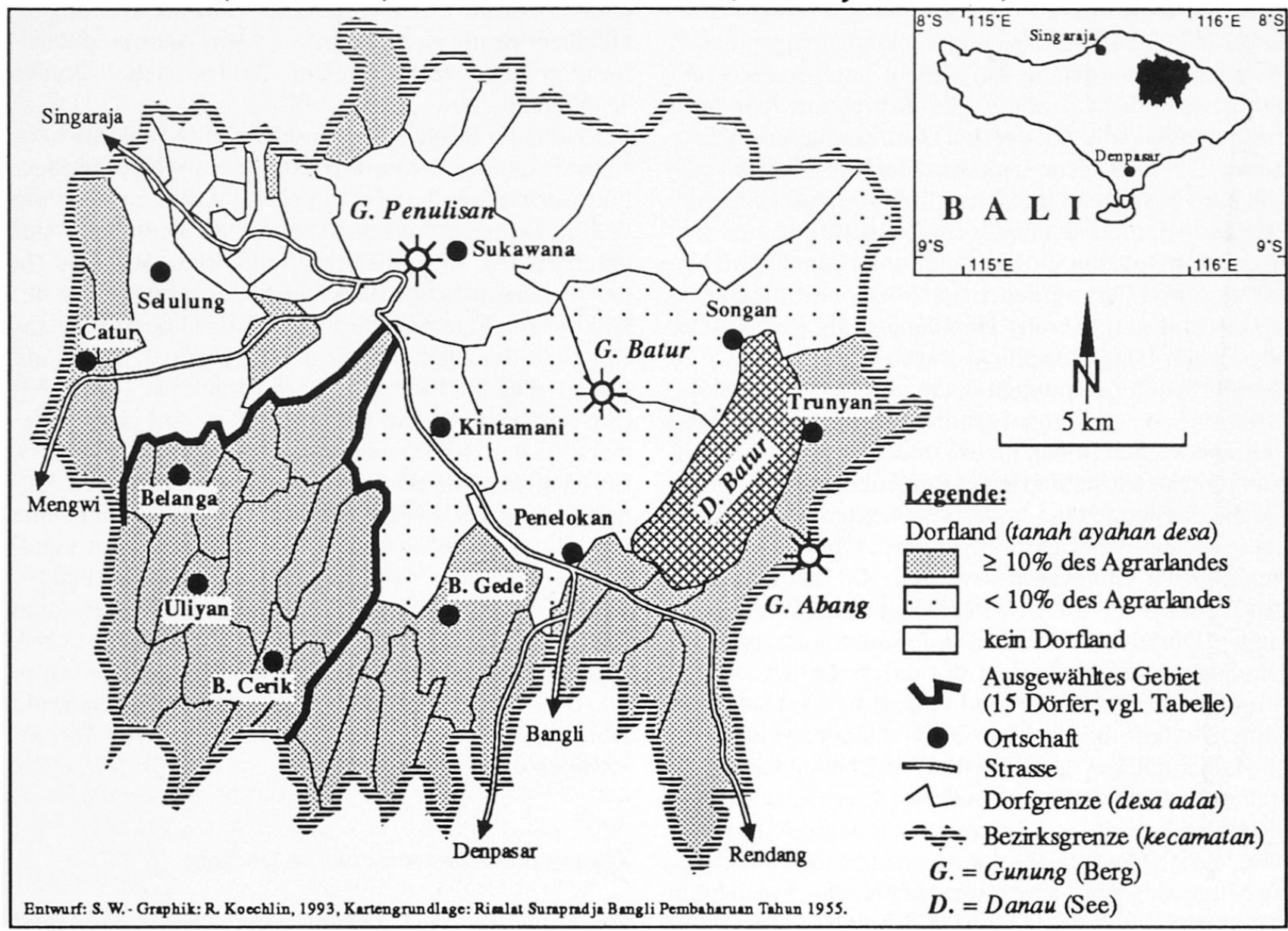

abgabe die einträglichste staatliche Einnahmequelle. Es erstaunt deshalb wenig, daß Volks- und Viehzählungen sowie Landvermessungen mit nordeuropäischer Gründlichkeit durchgeführt worden sind. Für den Bezirk Kintamani liefert nach wie vor einzig der Landsrente-(Landsteuer-)Bericht aus dem Jahr 1935 einige quantitative Angaben zu den Landeigentumsformen. Die Weltwirtschaftskrise der 30er Jahre brachte den Kaffee-Export zum Erliegen. Die ökonomische Lage blieb auch in den folgenden Jahrzehnten meist prekär. Nach einer leichten Erholung in den 50er Jahren bewirkte der Aschenregen nach dem Ausbruch des Vulkans Agung (1963) und die Periode des politischen Umsturzes (1966/67) eine schwere ökonomische Krise. Seit ungefähr 1975 ist ein wirtschaftlicher Aufschwung spürbar. Die steigende Nachfrage der neu entstehenden urbanen Mittel- und Beamtenschicht und Exportförderungsmaßnahmen der Regierung führten zu einem erneuten Anbau von cash crops. Neben Kaffee werden in kleinbäuerlichen Betrieben nun Vanille, Nelken, Orangen, Mandarinen und Limonen produziert. Acker- und Weideflächen werden teils in Baumgärten umgewandelt. Dies wirkt sich nicht nur auf die Physiognomie der Landschaft aus, sondern beein- flußt, wie weiter unten ausgeführt wird, auch die Institution Gemeinschaftsland.

\section{Gemeinschaftsland und Ältestenrat in Kintamani}

Die für die bali aga als typisch erachteten Institutionen sind an die traditionellen Gemeinden (desa adat) gebunden. Ein traditionelles Dorf umfaßt die bebauten und unbebauten Flächen innerhalb der Dorfgrenze. Die Dorfbewohner bilden eine Verehrungsgemeinschaft, welche ihren religiösen Verpflichtungen in drei Dorftempeln nachkommt: dem Ursprungstempel (pura puseh), dem Dorftempel (pura desa oder pura bale agung) und dem Tempel der Niederwelt bzw. des Todes (pura dalem). Die Dorfgemeinschaft verfügt zudem über das dorfeigene Land und überwacht die Einhaltung tradierter Werte und Normen. Schon diese sehr verkürzte Beschreibung ${ }^{1 "}$ verdeutlicht, daß ein desa adat ein komplexes, mehrdimensionales Gebilde ist, aus dem im folgenden nur wenige Elemente und Beziehungen herausgegriffen werden. Insbesondere der religiös-magische Bereich wird dabei vernachlässigt. 
Idealtypisch erhält in den Gemeinden des Bezirks Kintamani ein Dorfmitglied oder Dorfbürger (krama desa) eine Parzelle dorfeigenes Land zur landwirtschaftlichen Nutzung zugesprochen. Auf diesem Land (tanah) ruht eine Dienstpflicht (ayahan), die im wesentlichen darin besteht, zum Unterhalt der drei Dorf( desa)tempel beizutragen. Das dorfeigene Land wird deshalb als tanah ayahan desa bezeichnet und hier kurz Dorfland genannt. ${ }^{12}$ Der Tempelunterhalt umfaßt eine religiöse und eine physische Komponente, die hier beide unter dem Begriff Unterhalt subsumiert werden. Erstere beinhaltet die korrekte Durchführung sakraler Handlungen. Im Rahmen der physischen Unterhaltspflicht tragen die Dorfbürger zu den jährlichen Zeremonien sowie zur Erstellung und Renovation von zum Tempel gehörenden Bauten bei. Diese Beiträge werden in bar, natura und/oder als Arbeitsleistung entrichtet. Sie sind in der Regel unabhängig von der Fläche des genutzten Landes und werden pro Dorfbürger festgelegt. Jeder Dorfbürger nimmt an den Dorfversammlungen (sangkepan desa) teil, die ihrem vorwiegend rituellen Charakter entsprechend bei Vollmond, in einigen Dörfern auch bei Voll- und Neumond stattfinden. Die Sitzordnung entspricht der durch das Senioritätsprinzip bestimmten Rangordnung, d.h. der Dauer der Mitgliedschaft, die meist mit der Verheiratung eines Mitglieds beginnt. Die ranghöchsten Bürger bilden den Ältestenrat und verrichten entsprechend ihrem Rang bei rituellen Handlungen und Zeremonien festgelegte Funktionen. Je nach Dorf umfaßt der Ältestenrat sechs bis zwanzig Mitglieder, meist aber etwa acht bis zehn. Entsteht im Ältestenrat durch Rücktritt oder Tod eine Lücke, rücken alle rangniedrigeren Dorfbürger eine Position nach oben. So hat jeder Dorfbürger, ein langes Leben vorausgesetzt, die Möglichkeit, Mitglied des Ältestenrates zu werden, worin sich der egalitäre Charakter dieser Institution manifestiert.

Einen Ältestenrat kennen im Bezirk Kintamani 49 der 53 traditionellen Gemeinden. Die Absenz dieser für die bali aga typischen Institution läßt sich bei den übrigen vier Dörfern aus hier nicht näher erläuterten Aspekten ihrer Entwicklungsgeschichte ableiten. Weniger eindeutig ist die Sachlage beim Gemeinschaftsland, zu dem neben dem besprochenen Dorfland in einem weiteren Sinn auch das Tempelland (tanah laba pura) gehört. Tempelland wird üblicherweise von Teilbauern bewirtschaftet und in Kintamani mit Ausnahme eines Dorfes den Dorfbürgern nicht im Nutzrecht zugeteilt. Als Gemeinschaftsland im engeren Sinne wird deshalb hier das Dorfland bezeichnet und besprochen. ${ }^{13}$

Für den Bezirk Kintamani mit einer Gesamtfläche von 36692 ha weist die Arealstatistik 1984 landwirtschaftlich genutzte Flächen im Umfang von 22254 ha aus. Dabei werden Landnutzungsarten unterschieden, nicht aber Eigentumsformen. Dazu muß auf die Landvermessung des Jahres 1933 zurückgegriffen werden, die insofern Rückschlüsse erlaubt, als die landwirtschaftlich genutzte Fläche seither nur um wenige hundert Hektaren zugenommen hat. Von 19812 ha steuerpflichtigem land- wirtschaftlich genutztem Land waren 193314628 ha oder $73 \%$ in individuellem und 5184 ha oder $27 \%$ in kommunalem Besitz (tanah ayahan). Dem Agrarland zuzurechnen sind weiter ungefähr 2000 ha steuerbefreites Tempelland..$^{14}$

Individueller Besitz war also schon damals die vorherrschende Landeigentumsform. Immerhin wird die Bedeutung dorfeigenen Landes mit einem Anteil von 27\% am steuerpflichtigen Agrarland im Bezirk Kintamani klar unterstrichen. Ein differenzierteres Bild zeigt eine (in den niederländischen Berichten leider nicht enthaltene) Analyse der Eigentumsformen auf der Gemeindeebene. Die 1986 durchgeführte Erhebung ergab, daß etwa ein Drittel aller traditionellen Dörfer (nämlich 17 von 53) kein dorfeigenes Land besitzen und in drei großen Gemeinden der Dorflandanteil sehr gering ist (vgl. Karte). Ein Vergleich mit einer 1925 erfolgten qualitativen Aufnahme zeigt, daß in den dazumal erfaßten acht der heute siebzehn Gemeinden ohne Dorfland kommunaler Landbesitz schon damals seit langem unbekannt war und somit ein rezenter Wandel ausgeschlossen werden kann (vgl. GUNNING/VAN DER HEYDEN 1926: 343 f.). Im Bezirk Kintamani ist der Ältestenrat eindeutig eine Basisinstitution innerhalb der altbalinesischen Sozialordnung; beim Dorfland ist dies bei einer starken Minderheit der Gemeinden nicht der Fall.

\section{Zugang zum Ältestenrat und zu Dorfland}

Um die den baliaga-Gemeinden zugeschriebene Egalität näher auszuleuchten, muß eine bisher ausgeklammerte Frage gestellt werden: Wer wird Dorfbürger (krama desa) und erhält damit Zugang zum Ältestenrat und zur Bewirtschaftung dorfeigener Parzellen? In Dörfern ohne Dorfland beginnt die Vollmitgliedschaft in der Dorfgemeinschaft mit der Verheiratung. In den Gemeinden mit Dorfland hingegen geht die volle Dorfmitgliedschaft vom Vater auf nur einen Sohn über. Dieser übernimmt die Dorflandparzelle seines Vaters und hat damit auch zum Unterhalt der drei Dorftempel beizutragen. Alle anderen Familienvorsteher sind nicht vollberechtigte Bürger und werden meist als krama banjar bezeichnet. ${ }^{15}$ Diese erhalten höchstens eine kleine Hausparzelle aus dem Dorfland, aber kein Agrarland; sie sind nicht Mitglied der Gemeindeversammlung und können somit auch nie zum Ältestenrat aufrücken. Andererseits sind die krama banjar nur für einen der drei Dorftempel unterhaltspflichtig, den Tempel des Todes (pura dalem), der von allen Einwohnern (sozusagen der Einwohnergemeinde) gemeinsam unterhalten wird. ${ }^{16}$

Die Unterteilung der Bewohner in Dorfbürger und andere Einwohner belegt, daß zumindest die Gemeinden mit dorfeigenem Land eine geschichtete Sozialstruktur aufweisen. Anhand einer Gruppe von 15 Gemeinden soll die Bedeutung dieser Schichtung gezeigt werden. Diese Dörfer bilden eine räumlich homogene Einheit südwestlich der Baturcaldera und repräsentieren den Typus der 
Tab.: Dorfbürger und Gemeinschaftsland am Beispiel von 15 Dörfern des Bezirks Kintamani (1986)

\begin{tabular}{|c|c|c|c|c|c|c|c|c|}
\hline \multirow[t]{3}{*}{ Dorf } & \multicolumn{3}{|c|}{ Familienvorsteher } & \multicolumn{5}{|c|}{ Landwirtschaftliche Nutzfläche } \\
\hline & \multirow[t]{2}{*}{ Total } & \multirow{2}{*}{\multicolumn{2}{|c|}{$\begin{array}{l}\text { Dorfbürger } \\
\text { (krama desa) } \\
\text { in } \%\end{array}$}} & \multirow{2}{*}{$\begin{array}{l}\text { Total } \\
\text { ha }\end{array}$} & \multicolumn{2}{|c|}{$\begin{array}{c}\text { in Dorfbesitz } \\
\text { (t. ayahan desa) }\end{array}$} & \multicolumn{2}{|c|}{$\begin{array}{r}\text { in Tempelbesitz } \\
\text { (t. laba pura) }\end{array}$} \\
\hline & & & & & ha & in \% & ha & in $\%$ \\
\hline Belancan & 265 & 80 & 30 & 802 & 158 & 20 & 3 & 1 \\
\hline Mangguh & 100 & 30 & 30 & 164 & 30 & 18 & 1 & 1 \\
\hline Bayung Cerik & 119 & 35 & 29 & 215 & 51 & 24 & 0 & 0 \\
\hline Manikliyu & 296 & 140 & 47 & 435 & 133 & 31 & 0 & 0 \\
\hline Lembeyan & 126 & 75 & 60 & 276 & 75 & 27 & 6 & 2 \\
\hline Langgahan & 172 & 54 & 31 & 275 & 54 & 20 & 5 & 2 \\
\hline Serai & 141 & 125 & 89 & 373 & 85 & 23 & 20 & 5 \\
\hline Awan & 156 & 55 & 35 & 328 & 131 & 40 & 11 & 3 \\
\hline Gunungbau & 88 & 45 & 51 & 152 & 77 & 50 & 0 & 0 \\
\hline Uliyan & 124 & 56 & 45 & 284 & 45 & 16 & 0 & 0 \\
\hline Bunutin & 145 & 41 & 28 & 189 & 40 & 21 & 6 & 3 \\
\hline Belanga & 89 & 89 & 100 & 242 & 171 & 71 & 40 & 17 \\
\hline Batukaang & 119 & 70 & 59 & 158 & 88 & 55 & 5 & 3 \\
\hline Binyan & 62 & 30 & 48 & 119 & 103 & 85 & 13 & 11 \\
\hline Mengani & 140 & 59 & 42 & 387 & 202 & 52 & 10 & 4 \\
\hline Total & 2142 & 984 & 46 & 4399 & 1453 & 33 & 120 & 3 \\
\hline
\end{tabular}

Quelle: Eigene Erhebungen; Nutzfläche Total aus KANTOR STATISTIK PROPINSI BALI (1985)

kleineren Gemeinden mit dorfeigenem Land (vgl. Karte und Tab.). In den ausgewählten Gemeinden sind insgesamt $46 \%$ aller verheirateten Familienvorsteher auch Dorfbürger (krama desa). Die Variationsbreite erstreckt sich von $28 \%$ bis $100 \%$, wobei der Anteil der Bürger nur in zwei Dörfern zwei Drittel übersteigt. Nichtbürgern fällt es schwerer, einen hohen sozialen Status in der Gemeinde zu erreichen, im rituellen Bereich ist dies sogar mehr oder weniger ausgeschlossen. Eine Beurteilung dieser Benachteiligung aus der Sicht der Betroffenen kann hier nicht erfolgen, außer daß sie sich nicht direkt auf die ökonomische Lage der Haushalte auswirkt. Anders verhält es sich beim Dorfland. Die Bedeutung des Zugangs zum Dorfland ist abhängig von dessen Anteil am in der Gemeinde verfügbaren Agrarland. Aus der Tabelle wird ersichtlich, daß dieser Anteil in den untersuchten Gemeinden von $16 \%$ bis zu $85 \%$ variiert. Die im Nutzrecht an die Dorfbürger vergebenen Parzellen sind meist ausreichend groß, um den minimalen Unterhalt einer Familie zu sichern. Ist der Zugang zu Dorfland versperrt, so bleibt in einigen Fällen nur die Auswanderung. In mindestens drei der 15 Dörfer sind in den 80er Jahren Familien im Rahmen der staatlichen Transmigrationsprogramme nach Sulawesi übersiedelt. Häufiger aber ist, daß institutionelle Ungleichheiten durch informelle Vereinbarungen in der Familie gemildert oder ausgeglichen werden. Solche Formen der Anpassung werden aber mit zunehmender Landknappheit immer schwieriger.

\section{Dorfland und sozialer Wandel}

Eine diachrone Betrachtung zeigt, daß die altbalinesischen Institutionen Ältestenrat und im Nutzrecht an Dorfbürger vergebenes Dorfland nicht statisch sind, sondern durch ökonomische, politische und kulturelle Faktoren beeinflußt und verändert werden. Einige Aspekte dieses sozialen Wandels sollen anhand des Dorflandes gezeigt werden. 
Global (POPKIN 1979: 32 ff.), für Indonesien (SUDIYAT 1981: 2 ff.) und für Kintamani (GUNING/VAN DER HEYDEN 1926: 341 ff.) wird postuliert, daß mit zunehmender wirtschaftlicher Entwicklung und staatlicher Einwirkung Gemeinschaftsland in Individualbesitz übergeführt werde. Für Kintamani wird diese Transformation folgendermaßen beschrieben: Ursprünglich wird das Dorfland jährlich neu im Nutzrecht an die Berechtigten verteilt. In einem langsamen Prozeß werden dann die jährlichen Verteilungen durch periodische abgelöst. In der nächsten Phase ist das Nutzrecht für eine Parzelle in einer Familie vererblich, und schließlich geht das Land in deren Privateigentum über. Für alle diese Phasen führen GUNING/VAN DER HEYDEN Beispieldörfer aus der Region Kintamani an. Trotzdem erscheint die postulierte Entwicklung zu schematisch und gradlinig. Denn wie ließe sich damit erklären, daß in einer doch kleinen Region wie Kintamani verschiedene Phasen gleichzeitig anzutreffen sind, ja es sogar Dörfer gibt, wo bereits in den 20er Jahren seit Menschengedenken Dorfland unbekannt war, in anderen hingegen bis in die 70er Jahre jährliche Neuzuteilungen erfolgten.

Klarer scheint hingegen der Zusammenhang zwischen ökonomischen Faktoren und der Aufgabe der jährlichen Neuzuteilung von Dorfland. Eine jährliche oder auch nur periodische Zuteilung an die Nutznießer ist nicht kompatibel mit modernen Formen der Landnutzung. Zum einen wirkt sich - vor allem seit dem Verzicht auf Bracheperioden - die individuelle Nutzenmaximierung negativ auf die nachhaltige Erhaltung der Ertragskraft des Bodens aus. Einer der Dorfältesten von Bayung Gede, derjenigen Gemeinde, die 1979 als letzte in Kintamani die jährliche Neuverteilung abschaffte, drückt diesen Sachverhalt so aus: "... für eine gute Bewirtschaftung des Bodens ist es nicht möglich, daß heute gut bearbeitet wird, und nachher kriegt ein anderer das Land. Aber nun (nach der Aufgabe der jährlichen Neuverteilung) ist es nicht mehr so, jetzt wird das Land kontinuierlich bearbeitet, denn die Nachfolger sind immer die eigenen Nachkommen.» ${ }^{17}$ Zum andern verhindert eine jährliche Neuverteilung den Wechsel vom Anbau saisonaler Nutzpflanzen wie Trockenreis, Mais oder Maniok zu lukrativeren perennierenden Baum- und Strauchkulturen für die Produktion von Kaffee, Vanille, Nelken, Orangen und Mandarinen.

Aus (land)wirtschaftlicher Sicht ist die Ersetzung der jährlichen Zuteilung von Agrarlandparzellen durch ein quasi vererbbares Nutzungsrecht ausreichend. Die politischen Entwicklungen dieses Jahrhunderts hingegen fördern den Übergang zum Individualeigentum. Die 1925 von der Kolonialverwaltung eingeführte Landsteuer auferlegte die Steuerpflicht den direkten Nutzniessern der Dorflandparzellen. Zu den indonesischen Anstrengungen, die erdölunabhängigen Staatseinnahmen zu erhöhen, gehört die 1986 total revidierte Land- und Gebäudesteuer (pajak bumi dan bangunan, früher IPEDA). In diesem Kontext besteht die klare Absicht, gestützt auf das indonesische Agrargesetz aus dem Jahre 1960, indi- viduell genutzte Dorflandparzellen vom Nutzrecht ins Eigentumsrecht überzuführen (vgl. SUASTHAWA 1987: $43 \mathrm{ff}$.). Allerdings wird die Implementierung dieser Maßnahme in einer eher peripheren Region wie Kintamani wegen des damit verbundenen administrativen Aufwandes wahrscheinlich erst mittelfristig möglich sein. Eine solche Umwandlung kann durchaus im Interesse betroffener Bauern sein. So wurde schon in den 20er Jahren in relativ landarmen Dörfern von Kintamani die Tendenz festgestellt, periodische Neuverteilungen hinauszuzögern (GUNING/VAN DER HEYDEN 1926: 345 ).

Eher auf der kulturellen Ebene ist ein Spannungsfeld angesiedelt, welches die Institution des Dorflandes ebenfalls berührt. In den letzten beiden Jahrzehnten ist ein ausgeprägtes Bedürfnis entstanden, die ursprünglich einfachen Tempel in den Berggebieten baulich aufzuwerten und so in ihrer Erscheinung denjenigen des Tieflandes anzugleichen. Die Finanzierung dieser Vorhaben bedingt, daß entweder die Dorfbürger verstärkt zur Kasse gebeten werden, oder daß der Anteil der Bürger erhöht wird, was traditionell mit der Abgabe von Dorfland an die neuen Bürger verbunden ist. Dies wiederum liegt nicht im Interesse der bisherigen Dorfbürger, die nicht geneigt sind, auf Teile des von ihnen genutzten Landes zu verzichten. Welche Strategien in dieser Situation verfolgt werden, konnte in acht der fünfzehn oben diskutierten Dörfern anhand der Entwicklung der Zahl der Dorfbürger seit etwa 1930 erhoben werden. Es lassen sich drei generelle Strategien unterscheiden, die hier als demokratische, aristokratische und pragmatische bezeichnet werden. Diese werden anhand je eines Dorfbeispiels kurz vorgestellt, wobei die erste nur im besprochenen Dorf vorkommt, die anderen beiden etwa gleich häufig vertreten sind.

In der Gemeinde Belanga wird der demokratische Weg gewählt. Alle Verheirateten sind Dorfbürger. Im Nutzrecht bewirtschaftetes Land wird zu gleichen Teilen an alle verheirateten Söhne weitervergeben. Rechte und Pflichten sind somit in Belanga einigermaßen gleichmäBig verteilt. Anzufügen ist, daß diese demokratische Strategie in Zusammenhang mit der Tatsache steht, daß in Belanga nur 14\% des Agrarlandes in Individualbesitz sind (vgl. Tab.). Eher aristokratisch ist das Vorgehen in Bayung Cerik, wo der Dorfanteil am Agrarland allerdings bloß 24\% beträgt. Die Zahl der Dorfbürger wurde letztmals in den 30er Jahren von 26 auf 35 erhöht, und zwar durch ein Verfahren, das als für die bisherigen Bürger "schmerzlose» Landvergabe bezeichnet werden kann. Denn das gesamte Tempelland wurde aufgelöst und als Dorfland im Nutzrecht an die neuen Bürger verteilt. Seither hat sich die Bevölkerung verdreifacht, die Zahl der Bürger ist hingegen konstant geblieben. 1984 beschloß die Gemeinde, die Pflichten zu demokratisieren - nicht aber den Zugang zum Dorfland. Seither haben alle Familien zum Unterhalt aller drei Dorftempel beizutragen. Es dürfte interessant sein, die Folgewirkungen dieser durchaus einseitigen Anpassung weiter zu be- 
obachten. In der 1930 keine hundert Einwohner zählenden Gemeinde Uliyan wurde bis etwa 1945 Dorfland sogar an Neuzuzüger vergeben und diese als Dorfbürger aufgenommen, um so den Tempelunterhalt in dieser kleinen Gemeinde zu gewährleisten. Seither wird eine pragmatische Strategie verfolgt. Die Zahl der Bürger wird erhöht, wenn dies ohne Beeinträchtigung der Nutzungsrechte der bisherigen Bürger möglich ist. Die Gemeinde hat seit 1950 freiwerdendes Amtsland und (aufgrund gesetzlicher Bestimmungen über maximal erlaubte Betriebsflächen) eingezogene Grundstücke sowie Tempelland in Dorfland überführt und an rund 20 neue Bürger verteilt. Zudem ist der auch in anderen Dörfern bekannte Brauch, daß Kranke geloben, bei ihrer Genesung die Dorftempel zu unterstützen, in Uliyan besonders stark verbreitet. Der Nutzen, d. h. die Wiedergenesung, wird damit vorbezogen; die in der Folge anfallenden Verpflichtungen stärken die rituelle Sphäre der Dorfgemeinschaft.

Die drei unterschiedlichen Strategien weisen zwei Gemeinsamkeiten auf: Der Unterhalt der drei Dorftempel wird sichergestellt, ohne daß den bisherigen NutznieBern Land zur Neuverteilung (ausgenommen innerhalb der eigenen Familie in Belanga) entzogen wird. Zudem wird der innere Zusammenhang von Dienstpflichten gegenüber den drei Dorftempeln einerseits und den Nutzrechten an kommunalen Landparzellen andererseits durch alle Strategien tendenziell gelockert.

\section{Diskussion}

In der Mehrheit der untersuchten bali aga-Dörfer im Bezirk Kintamani besteht weder zur Nutzung von Land in kommunalem Besitz noch zur Gemeindeversammlung und damit dem Ältestenrat ein egalitärer Zugang. Denn dieser bleibt den Dorfbürgern, den krama desa, vorbehalten. In den eingehender besprochenen 15 Gemeinden sind dies etwas weniger als die Hälfte der Familienvorsteher. Zudem werden in etlichen Dörfern führende Positionen in den traditionellen und administrativen Gemeinden von einer oder mehreren Familien permanent besetzt, die Ämter quasi in der Familie vererbt. Andererseits entzieht die Institution des Dorflandes dem Markt Land und verhindert so die Entstehung krasser Gegensätze in der Landbesitzverteilung.

Die als für die bali aga typisch erachtete Institution des dorfeigenen Agrarlandes, das im Nutzrecht an die berechtigten Bürger vergeben wird, ist in einem Drittel der 53 traditionellen Gemeinden Kintamanis unbekannt. In den anderen Gemeinden begünstigten wirtschaftliche Faktoren die Abkehr von jährlichen oder periodischen Landverteilungen. Das staatliche Steuer- und Rechtssystem fördert den Übergang zum Individualeigentum. Den einzelnen Gemeinden verbleibt zumindest mittelfristig das Verfügungsrecht über das Dorfland. Faktisch hat sich aber das vererbbare Nutzrecht an dorfeigenen Landparzellen durchgesetzt, so daß mit verschiedenen Strate- gien versucht wird, entweder die Zahl der Dorfbürger zu erhöhen oder die früher den dorflandberechtigten Bürgern auferlegte Aufgabe, die drei Dorftempel zu unterstützen, auf alle Einwohner auszudehnen.

Empirische Erhebungen auf der Gemeindeebene vermitteln damit ein anderes und weniger egalitäres Bild als allgemeine Darstellungen der Sozialordnung der bali aga. Andererseits ist es vor allem für die bisherige kulturanthropologische Auseinandersetzung mit Kultur und Gesellschaft der bali aga bezeichnend (bspw. HOWE 1989 und vor allem LANSING 1983), daß nicht nur die durchaus vorhandenen egalitären Elemente der bali aga überbewertet werden, sondern auch die für die eher - aber nicht ausschließlich - profaneren Aspekte des Lebens zuständigen Gremien und Ämter innerhalb der traditionellen und administrativen Gemeinden vernachlässigt werden. Pointiert ausgedrückt wird manchmal der Eindruck erweckt, als ob sich Bali noch in der vorkolonialen Fürstenzeit befinde. Es ist wohl Ansichtssache, welcher Institution die größere Bedeutung beigemessen wird, Tatsache ist aber, daß beide existieren: der Ältestenrat - wo schon durch die Rangordnung, ausgedrückt auch in der Sitzordnung an Zeremonien und durch die rituelle Bedeutsamkeit der ausgeführten Handlungen sowie durch das Prinzip des automatischen Nachrückens eine kosmische Ordnung, der zyklische Charakter der Zeit, die immerwährende Wiederkehr des Gleichen ausgedrückt wird wie auch die Gremien und Ämter der traditionellen und administrativen Gemeinden, die sich mit Geld, Organisation und Bauführung, dem linearen Aspekt der Zeit befassen.

Für andere Gebiete Balis wurde in neueren Arbeiten am Beispiel von Basisinstitutionen wie auch von Ritualen gezeigt, daß sich gerade die dynamischen Aspekte, der soziale Wandel in der Auseinandersetzung zwischen Staat und Gemeinde manifestieren (SCHULTE NORDHOLT 1991, TARNUTZER 1993, wARREN 1990). Die Auswirkungen des Kolonialismus, die Ausdehnung der Marktwirtschaft, sowie die Entstehung von Nationalstaaten verändern global ehemals vorkapitalistische ländliche Gesellschaften (vgl. POPKIN 1979). Auch in Kintamani sind die Auswirkungen wirtschaftlicher, nationaler, ja sogar panbalinesischer Integration spürbar. ${ }^{18}$ Dies wird weitere Anpassungsprozesse der bali aga von Kintamani erfordern. Damit dabei die kulturelle Identität erhalten bleibt, ist eine relativ nüchterne Bestandesaufnahme und Bewertung der bali aga-Kultur und Sozialordnung notwendig.

\section{Anmerkungen}

'Entsprechende Schilderungen sind in vielen Reisehandbüchern enthalten, beispielsweise in DALTON (1985: 461): "Armut wird groß geschrieben in dieser Bergregion Balis. Aber möglicherweise leben in der Umgebung von Penelokan (Aussichtspunkt auf der Baturcaldera, vgl. Karte, sw) auch die aggressivsten und geldgierigsten Menschen von ganz Indonesien... Da die Bewohner dieser Gegend sehr abgeschieden leben, ist Inzucht unverkennbar. Nicht immer 
sind diese Menschen schön anzusehen. Sie können Fremden einen tüchtigen Schrecken einjagen. Ehrenwert sind sie nicht gerade, eher ein wenig bösartig." Nicht nur Autoren von Reisehandbüchern, sondern oft auch Balinesen betrachten die bali aga als in jeder Beziehung rückständig.

${ }^{2}$ Die Forschungen von Margaret Mead befaßten sich unter anderem mit kulturellen Aspekten der Schizophrenie, mit der Frage, wie auf Bali die normale Sozialisation Individuen hervorbringe, die im Westen als schizoid bezeichnet würden Bezeichnenderweise wurde die Forschung finanziert vom American Museum of Natural History, die Publikation vom Research Committee for Research in Dementia Praecox (HOWARD 1985: 181). Eine kritische Auseinandersetzung mit der wissenschaftlichen Arbeit von Margaret Mead und ihrem Forscherehemann Gregory Bateson in Bali liefern JENSEN/SURYANI (1992), die Bateson und Mead andererseits bewundern für ihre Fähigkeit, in Bayung Gede zu wohnen, einem Dorf, "where life is obviously hard, dirty, and relatively unhealthy"(!) (S. 11)

${ }^{3}$ Diese Auffassungen vertreten HOWE (1989), SCHAAREMAN (1986), WARREN (1990).

${ }^{4}$ Auch Margaret Mead and Gregory Bateson sollen in ihrem Untersuchungsdorf Bayung Gede nur wenige Spuren des Hinduismus angetroffen haben (JENSEN/SURYANI 1992 $36 \mathrm{ff}$.).

${ }^{5}$ Beiträge an den Forschungsaufenthalt gewährten die Stiftung für wissenschaftliche Forschung an der Universität Zürich, die Jubiläumsspende für die Universität Zürich und die STEO-Stiftung Zürich.

Alle nicht zitierten Angaben beruhen auf persönlichen Interviews. Flächenangaben sind auf Plausibilität geprüft, aber kleinere Abweichungen in Kauf genommen worden, denn im Vordergrund stand nicht ein statistisches Interesse, sondern die Eruierung von Veränderungsprozessen. Da solche Prozesse teils konfliktträchtig sind, bestand in den Interviews die Tendenz, diese womöglich elegant zu umgehen. Die Zahl und Bedeutung solcher Prozesse wurde deshalb wohl eher unter- denn überschätzt.

${ }^{6}$ Vgl. SUKARTO (1987: 32), der noch andere, allerdings weniger wahrscheinliche Bedeutungen von cintamani diskutiert. ${ }^{7}$ Für Buahan vgl. GORIS (1954:176f.), für Sukawana WARDHA (1983:8f.)

${ }^{8}$ In den von GORIS (1954) zusammengestellten und numerierten Urkunden PB 001, 005, 007, 104, 108, 302 enthält die Liste vererbbarer Güter unter anderem verschiedene Landnutzungstypen.

${ }^{9}$ In den älteren Urkunden huma parlak padang mmal, heute sawah, tegal, padang, kebun. Als weitere Kategorie wird alas (Ur-)Wald erwähnt, aber nicht im Zusammenhang mit individuellen Rechten.

${ }^{10} \mathrm{Vgl}$. dazu den Artikel von A. Tarnutzer in dieser Nummer.

" Vgl. dazu ausführlicher den Artikel von A. Leemann in dieser Nummer.

${ }^{12}$ Oft sind die bebauten Gebiete (tanah pakarangan desa) ebenfalls im Besitz der Gemeinde.

${ }^{13}$ Der Vollständigkeit halber sei angeführt, daß etwa ein Viertel der Fläche von Kintamani im Besitz des Staates (tanah negara) ist. Diese hauptsächlich als Wald ausgewiesenen Flächen werden in der Arealstatistik den Gemeinden zugerechnet, das Verfügungsrecht liegt aber bei der Forstbehörde.

${ }^{14}$ Zusammengestellt aus Beilage 1B, S. 3f., und 82 der MONOGRAPHIE van der ONDERAFDEELING KLOENGKOENG (1935). Unter der Annahme, daß steuerbefreites Tempelland proportional zu den besteuerten Ländereien unter den vier
Bezirken von Bangli verteilt sind, entfallen rund 2000 ha auf Kintamani.

${ }^{15}$ Die Bedeutung des Begriffs banjar ist in bali aga-Gemeinschaft und solchen der Hochkultur nur teilweise deckungsgleich

${ }^{16}$ Unterschiedliche Rechte für Ortsbürger und andere Einwohner sind auch in der Schweiz nicht unbekannt.

17 Interviewtranskript Bayung Gede Oktober 1986.

${ }^{18}$ In WÄLTY (in Vorbereitung) wird diese Thematik ausführlicher dargestellt.

\section{Literatur}

DALTON, B. (1985): Indonesien-Handbuch, Bremen.

GEERTZ, C. (1959): Form and Variation in Balinese Village Structure. In: American Anthropologist, 6, 991-1012.

GEERTZ, C. (1980): Negara, the Theatre State in 19th Century Bali, Princeton.

GORIS, R. (1954): Prasasti Bali, Bandung.

GUNING, H. C.; van der HEYDEN, A. J. (1926): Het Petjatoeen Ambtsvelden Problem in Zuid-Bali. In: Tijdschrift voor Indische Taal-, Land- en Volkenkunde, 56 (3), 319-394.

HOWARD, J. (1985): Margaret Mead - a Life, New York.

JENSEN, G. D.; SURYANI, L. K. (1992): The Balinese People. A Reinvestigation of Character, Singapore.

KANTOR STATISTIK PROPINSI BALI (1985): Statistik Kecamatan Kintamani 1984, Denpasar.

KORN, V. E. (1932): Het Adatrecht van Bali, 's-Gravenhage.

LANSING, S. J. (1983): The Three Worlds of Bali, New York.

LEEMANN, A. (1976): Bali: Auswirkungen des balinesischen Weltbildes auf verschiedene Aspekte der Kulturlandschaft und auf die Wertung des Jahresablaufes, Bern.

MONOGRAPHIE VAN DER ONDERAFDEELING KLOENGKOENG (1935).

POPKIN, S. L. (1979): The Rational Peasant, Berkeley.

SCHAAREMAN, D. (1986): Tatulingga: Tradition and Continuity: An Investigation in Ritual and Social Organization in Bali, Basel.

SCHULTE NORDHOLT, H. (1991): State, Village, and Ritual in Bali, Amsterdam.

SUASTHAWA, M. D. (1987): Tanah adat Bali setelah berlakunya UUPA, Denpasar.

SUDIYAT, I. (1981): Hukum adat, Sketsa asas, Yogyakarta.

SUKARTO, K. A. (1987): Benda purbakala dari Pura Tulukbiyu di Bali. In: Berkela Arkeologi, 8(1), 31-45

TARNUTZER, A. (1993): Kota Adat Denpasar (Bali): Stadtentwicklung, staatliches Handeln und endogene Institutionen, Zürich.

WÄLTY, S. (in Vorb.): Kintamani: Dorf, Landbesitz und Feiertage. Institutionen im Entwicklungsprozeß in einer peripheren Region auf Bali, Zürich.

WARDHA, W. (1983): Perdagangan dan komoditi dalam jaman Bali Kuna (suatu kajian dari temuan 4 lembar prasasti d pura Desa Bale Agung, Kintamani). Paper delivered at Pertemuan IImiah Arkeologi III, Ciloto.

WARREN, C. (1990): Adat and Dinas: Village and State in Contemporary Bali, Perth. 